\title{
TECNOLOGIA EDUCACIONAL EM SAÚDE: CONTRIBUIÇÕES PARA O CUIDADO COM QUIMIOTERÁPICOS EM PEDIATRIA
}

\section{ARTIGO ORIGINAL}

MARTINS, Paulo Alexandre ${ }^{1}$

SCHERER, Juliane de Souza ${ }^{2}$

MESQUITA, Kelly ${ }^{3}$

PEREIRA, Jéssica Silveira ${ }^{4}$

ROST, Bruna Luisa ${ }^{5}$

VIANA, Marcele ${ }^{6}$

NEGELISKII, Christian ${ }^{7}$

\footnotetext{
${ }^{1}$ Acadêmico em Enfermagem pela Universidade Feevale, Novo Hamburgo, RS.

2 Professora Assistente do Curso de Enfermagem e do Programa de Pós-Graduação pela Universidade Feevale, Novo Hamburgo, RS. Doutoranda em Ciências da SaúdeCardiologia pelo PPG da Fundação Universitária de Cardiologia/RS.

${ }^{3}$ Mestre em Ensino na Saúde pelo PPGSENSAU da Universidade Federal de Ciências da Saúde de Porto Alegre, UFCSPA/RS. Professora Assistente do Curso de Graduação em Enfermagem da Faculdade de Desenvolvimento do Rio Grande do Sul, FADERGS/RS. Especialista em Enfermagem Pediátrica pela Faculdade Unyleya.

${ }^{4}$ Acadêmica em Enfermagem pela Universidade Feevale, Novo Hamburgo, RS.

${ }^{5}$ Acadêmica em Enfermagem pela Universidade Feevale, Novo Hamburgo, RS.

${ }^{6}$ Acadêmica em Enfermagem pela Universidade Feevale, Novo Hamburgo, RS.

7 Doutor em Enfermagem, Professor Adjunto do Curso de Enfermagem na Universidade Feevale, Novo Hamburgo, RS. Coordenador da Pós-graduação em Terapia intensiva adulto da Universidade Feevale.
} 
MARTINS, Paulo Alexandre. Et al. Tecnologia educacional em saúde: contribuições para o cuidado com quimioterápicos em pediatria. Revista Científica Multidisciplinar Núcleo do Conhecimento. Ano 05, Ed. 03, Vol. 05, pp. 130 140. Março de 2020. ISSN: 2448-0959, Link de acesso: https://www.nucleodoconhecimento.com.br/saude/tecnologia-educacionalem-saude

\section{RESUMO}

Os quimioterápicos antineoplásicos são cada vez mais utilizados no tratamento de neoplasias, e, por isso, é fundamental que o enfermeiro possua conhecimento sobre o manuseio desses fármacos. Dessa forma, a utilização de tecnologias inteligentes são iniciativas que podem contribuir para a promoção da segurança do paciente pediátrico hospitalizado. $\mathrm{O}$ objetivo geral deste estudo foi identificar as necessidades da ferramenta auxiliar tipo aplicativo como apoio para solucionar as dúvidas que possam ocorrer durante a administração de quimioterápicos em pediatria, com base em informações e necessidades citadas pelos enfermeiros, para identificar quais drogas foram sugeridas, suas particularidades durante a administração e para a resolução de eventos adversos ou intercorrências. A gama de medicamentos utilizados no tratamento oncológico é vasta e suas categorias variam de antibióticos a anticorpos monoclonais, sendo utilizados nos protocolos em combinação com as drogas mais tradicionais, tendo extrema importância para a efetividade do conjunto das combinações de medicamentos. Com base nessas pontuações, pode-se concluir que um instrumento que facilita o trabalho e cria um ambiente seguro no tratamento de pacientes pediátricos em tratamento oncológico é valido. Salienta-se, então, a importância de um aplicativo que possa auxiliar os profissionais no seu dia a dia de trabalho para este grupo de pacientes ou, ainda, de outras especialidades.

Palavras-chave: Enfermagem pediátrica, oncologia, quimioterapia, quimiotratamento, aplicativos móveis. 


\section{INTRODUÇÃO}

Os quimioterápicos antineoplásicos são utilizados isolados ou em combinação, sendo cada vez mais utilizados no tratamento de tumores malignos, tornando-se uma das mais importantes e promissoras formas de combate ao câncer. Para isso, é fundamental que o profissional de enfermagem possua o conhecimento técnico relacionado ao manuseio destes fármacos para que possa prestar informações corretas e esclarecedoras aos pacientes e familiares (MOLINA; MOTA, 2012). Para Wegner et al (2017), a utilização de tecnologias inteligentes e a padronização/protocolização das práticas são iniciativas que podem contribuir para a promoção da segurança do paciente pediátrico hospitalizado com repercussões diretas na atenção à saúde. Os equipamentos móveis, como telefones celulares que possuem maior capacidade de processamento de dados (smartphones) e os dispositivos portáteis (tablets) que possuem acesso rápido à internet podem contribuir para com esse processo.

Tais aplicativos, incluindo as informações geradas pelos mesmos, podem ser utilizados para otimização dos resultados e redução dos riscos na área da saúde bem como para compreensão dos fatores determinantes que promovem a saúde e/ou que levam à doença (BARRA et al, 2017). Tendo em vista as dificuldades e escassez de recursos físicos, humanos, estruturais e materiais, grande parcela dos serviços de saúde tornam a prática educativa monótona, desestimulante e repetitiva para o profissional. Acreditamos que os materiais de ensino dinamizam as atividades de Educação em Saúde e o uso de tecnologias na educação de profissionais da saúde tem sido uma prática muito disseminada entre estes.

Considerando este contexto, é válido ressaltar que existem algumas ferramentas disponíveis no mercado, entretanto elas nem sempre atendem às necessidades dos profissionais de enfermagem diante das situações ou eventos inesperados, pois são mais direcionadas a particularidades e especificidades médicas e algumas, ainda, são direcionadas para os pacientes. Este estudo foi desenvolvido após identificar as dificuldades encontradas pelos profissionais de enfermagem em razão da falta de

Disponível em: https://www.nucleodoconhecimento.com.br/saude/tecnologia-educacional-emsaude 
conhecimento sobre o tema e pelo difícil acesso aos conteúdos relacionados aos quimioterápicos.

\section{METODOLOGIA}

Trata-se de um estudo transversal, com abordagem quantitativa e descritiva, e conta com uma população composta por enfermeiros que trabalham em hospitais que atendem pacientes pediátricos na cidade de Porto Alegre e administram quimioterápicos em geral. Dessa forma, a amostra foi constituída pelos enfermeiros atuantes em oncologia pediátrica que aceitaram responder o questionário on line e atenderam aos critérios de inclusão e exclusão do proposto estudo. Totalizou-se 17 enfermeiros respondentes, caracterizando uma amostra por conveniência. Para a inclusão no estudo, o profissional deveria ter completado no mínimo 6 meses de experiência administrando tanto quimioterapia via oral como endovenosa no seu dia a dia de trabalho. Foi incluído o enfermeiro que concordou em participar do estudo por meio do aceite do Termo de Consentimento Livre e Esclarecido (TCLE) e aquele que respondeu o questionário enviado por e-mail.

Foram excluídos os profissionais que se negaram a participar do estudo ou não contemplaram os quesitos estabelecidos. A pesquisa foi desenvolvida seguindo os padrões éticos da Resolução CNS №466/2012 a qual considera que todo o progresso e seu avanço devem, sempre, respeitar a dignidade, a liberdade e a autonomia do ser humano. As informações foram recolhidas por meio de questionário desenvolvido com base nas situações relevantes e nas possíveis complicações envolvidas na administração de quimioterápicos em pediatria (APENDICE B). Este questionário foi reproduzido no Google Forms ${ }^{\circledast}$ e enviado aos participantes selecionados via e-mail. Os dados foram compilados em uma planilha obtida após a finalização da pesquisa no Google Forms ${ }^{\circledR}$, sendo analisados e expressos em frequência simples e absoluta e discutidos com as evidências científicas atuais. 


\section{APRESENTAÇÃO E ANÁLISE DOS DADOS}

O questionário foi respondido por 17 (dezessete) enfermeiros e enfermeiras que atuam em unidades pediátricas de hospitais de Porto Alegre. Ele apresenta informações como idade, tempo de formação e de atuação. Os respondentes têm idades que variam entre 24 e 56 anos de idade, sendo somente um enfermeiro, o restante dos respondentes foi de enfermeiras. O tempo de formação variou de 1 ano e 10 meses a 22 anos e o tempo de atuação como enfermeiros é de 1 ano e 7 meses a 22 anos. Os que trabalham exclusivamente com pacientes oncológicos são 35,3\%, e o restante $(64,7 \%)$ dos respondentes trabalham em unidades que internam pacientes com outras patologias não oncológicas. A tabela 1 ilustra os resultados:

Tabela 1 - Respostas dos enfermeiros pediátricos sobre o tempo de formação e o tempo de trabalho com oncologia pediátrica (Porto Alegre, março/2019)

\begin{tabular}{|c|c|c|c|c|}
\hline \multirow[t]{2}{*}{ Tempo } & \multicolumn{2}{|c|}{$\begin{array}{l}\text { Quanto tempo de } \\
\text { formação? }\end{array}$} & \multicolumn{2}{|c|}{$\begin{array}{l}\text { Quanto tempo trabalha com } \\
\text { oncologia pediátrica? }\end{array}$} \\
\hline & $N$ & $\%$ & $\mathrm{~N}$ & $\%$ \\
\hline $\begin{array}{l}\text { De } 1 \text { mês a } 5 \\
\text { anos }\end{array}$ & 9 & 52,95 & 12 & 70,59 \\
\hline De 6 a 10 anos & 4 & 23,53 & 2 & 11,77 \\
\hline De 11 a 15 anos & 2 & 11,76 & 1 & 5,88 \\
\hline De 16 a 20 anos & 0 & 0 & 1 & 5,88 \\
\hline Mais de 20 & 2 & 11,76 & 1 & 5,88 \\
\hline Total & 17 & $100 \%$ & 17 & $100 \%$ \\
\hline
\end{tabular}

Fonte: Pesquisa direta feita pelos autores (2019).

$64,7 \%$ dos enfermeiros trabalham em unidades mistas, nas quais a rotina de administração de quimioterápicos pode ser confundida ou gerar dúvidas em virtude de esta não ser uma prática diária. Assim, o grande volume de informações das mais variadas patologias que o profissional precisa ter conhecimento pode permitir

Disponível em: https://www.nucleodoconhecimento.com.br/saude/tecnologia-educacional-em$\underline{\text { saude }}$ 
confusões ou dúvidas. A maioria dos enfermeiros referiu que seu tempo de formação coincide com o tempo em que trabalham como enfermeiros e na oncologia pediátrica, ou seja, logo após a conclusão de curso, foram alocados em setores complexos que necessitam de experiência profissional. Chama atenção pois eles têm uma bagagem de conhecimento puramente acadêmica, bagagem esta que vai se aprimorar com os problemas encontrados no dia a dia de trabalho. A segurança e a qualidade do atendimento ao paciente estão vinculadas ao tempo de experiência do profissional que vai atendê-lo, porém, sabemos que outros fatores podem influenciar na boa assistência.

As condições em que este profissional está inserido podem influenciar diretamente, pois são seres humanos e passíveis à erros e vários problemas podem desestruturar este "ser humano" e causar algum desequilíbrio emocional que pode levar a uma má qualidade na prestação do atendimento (OLIVEIRA et al, 2014). Dos 17 respondentes, apenas 4 (quatro) não possuem algum tipo de especialização. Dos restantes, 4 (quatro) possuem pós-graduação em Enfermagem Oncológica; 3 (três) possuem Residência Multiprofissional em Atenção ao Câncer Infantil; 1 (um) pós-graduação em Urgência, Emergência e Uti; 1(um) Enfermagem Pediátrica e MBA em Gestão em Saúde; 1 (um) Especialização em Onco - Hematologia; 1(um) Enfermagem em Oncologia e Tratamento da Dor e Medicina Paliativa; 1 (um) pós-graduação em Emergência e UTI Pediátrica e 1 (um) em Terapia intensiva.

A seguir, o material pesquisado será apresentado a fim de proporcionar visibilidade aos resultados obtidos e evidenciar as necessidades expressadas nos mesmos. $\mathrm{Na}$ sequência, serão discutidos os dados obtidos. A tabela 2 relaciona o número de vezes que cada profissional manuseia quimioterápicos em uma semana de trabalho.

Tabela 2 - Respostas dos enfermeiros pediátricos sobre quantas vezes aproximadamente manuseiam quimioterápicos na semana (Porto Alegre, março/2019)
Variável
№ respostas
$\%$ total

Disponível em: https://www.nucleodoconhecimento.com.br/saude/tecnologia-educacional-em- 


\begin{tabular}{|l|l|l|}
\hline $\mathbf{0}$ a $\mathbf{2}$ vezes & 3 & 17,6 \\
\hline $\mathbf{3}$ a $\mathbf{4}$ & 3 & 17,6 \\
\hline $\mathbf{5}$ a $\mathbf{6}$ & 1 & 5,9 \\
\hline $\mathbf{7}$ a 8 & 2 & 11,8 \\
\hline $\mathbf{9}$ a $\mathbf{1 0}$ & 1 & 5,9 \\
\hline Acima de $\mathbf{1 0}$ & 7 & 41,2 \\
\hline Total & 17 & 100 \\
\hline
\end{tabular}

Fonte: Pesquisa direta feita pelos autores (2019).

A maioria dos enfermeiros que responderam à pesquisa manuseia os quimioterápicos diariamente $(41,7 \%)$, visto que referem trabalhar com tais produtos acima de 10 vezes durante a semana. Todavia, os outros profissionais fazem este manuseio em proporções menores durante o mesmo período, não estabelecendo uma rotina diária, fazendo com que as informações pertinentes possam ser esquecidas pelo profissional. Este é um tema delicado, pois o manuseio inadequado destes quimioterápicos pode causar uma infinidade de consequências tanto ao paciente quanto ao profissional que $o$ administra. $O$ processo de trabalho varia de acordo com o ambiente, resultante das problemáticas e do déficit do profissional, o que acarreta o impacto direto no atendimento e no desgaste do profissional e até mesmo ao meio ambiente (STEFFENS, 2011). As ferramentas educativas disponíveis, do tipo aplicativo, serão relatadas na tabela 3 , a seguir:

Tabela 3 - Respostas dos enfermeiros pediátricos sobre ferramentas educativas disponíveis sobre quimioterapia (Porto Alegre, março/2019)

\begin{tabular}{|l|l|l|l|l|l|}
\hline $\begin{array}{l}\text { Você conhece alguma ferramenta educativa } \\
\text { sobre administração de quimioterápicos }\end{array}$ & Não $\%$ & Total \\
$\begin{array}{l}\text { (aplicativo) que contenha informações sobre } \\
\text { cuidados na administração de } \\
\text { quimioterápicos? }\end{array}$ & 0 & 17 & 100 & 17 \\
\hline
\end{tabular}

Disponível em: https://www.nucleodoconhecimento.com.br/saude/tecnologia-educacional-em- 


\begin{tabular}{|l|l|l|l|l|l|}
\hline $\begin{array}{l}\text { Se conheces, esta ferramenta educativa } \\
\text { sobre administração de quimioterápicos } \\
\text { (aplicativo) contém informações úteis e }\end{array}$ & 0 & 17 & 100 & 17 \\
$\begin{array}{l}\text { relevantes para o seu dia a dia de trabalho? } \\
\text { Você sente necessidade da criação de uma } \\
\text { ferramenta educativa sobre administração de } \\
\text { quimioterápicos (aplicativo) com orientações } \\
\text { sobre cuidados na administração de } \\
\text { quimioterápicos? }\end{array}$ & 0 & 17 & 00 & 17 \\
\hline
\end{tabular}

Fonte: Pesquisa direta feita pelos autores (2019).

Assim sendo, ao considerar e analisar esses dados, podemos observar a necessidade da criação de uma ferramenta educativa sobre a administração de quimioterápicos relatada pelos profissionais que responderam ao questionário. Ela facilitaria a prática diária dos profissionais e minimizaria os problemas aqui identificados. Eles desconhecem a existência de algo que pudesse suprir suas necessidades de informação de uma forma mais rápida e prática, ou seja, em forma de aplicativo para celular. As atualizações na área da saúde acontecem frequentemente, junto a isso, há uma diversidade de patologias atendidas, e, assim, a falta da prática diária na administração de quimioterápicos somente reforça a necessidade de conhecimento sobre as especificidades das drogas. A resolução RDC № 220, de 21 de setembro de 2004, aprova o regulamento técnico para os Serviços de Quimioterapia, e, nesse sentido, precisa ser considerada.

Esta estabelece que o profissional que trabalha com quimioterápicos precisa ter treinamento inicial e continuado para sua capacitação efetiva na prestação do atendimento (BRASIL, 2004). Entre os anos de 2007 e 2008 aconteceram diversos fóruns de debates que identificaram a necessidade de uma política nacional que orientasse a avaliação, a incorporação e a gestão de tecnologias em saúde, culminando na aprovação, em 2009, pelo Conselho Nacional de Saúde (CNS) e pela Comissão Inter gestores Tripartite (CIT) com a publicação da Portaria no 2.690, de 5 
de novembro de 2009, que institui a Política Nacional de Gestão de Tecnologias em Saúde. O objetivo desta política é o de garantir que as tecnologias sejam seguras e eficazes bem como promove o conhecimento técnico-científico (BRASIL, 2010). A tabela 4 apresenta quais tópicos são relevantes para apoiar a práticas dos enfermeiros na administração de quimioterápicos para o público pediátrico:

Tabela 4 - Respostas dos enfermeiros pediátricos sobre quais tópicos são mais importantes constar em uma ferramenta educativa (aplicativo) (Porto Alegre, março/2019)

São tópicos necessários em uma ferramenta educativa sobre administração de quimioterápicos (aplicativo), informações como:

\begin{tabular}{|c|c|c|}
\hline Variáveis* & № respostas & $\%$ total \\
\hline Tempo de estabilidade após preparo & 15 & 88,2 \\
\hline $\begin{array}{l}\text { Pré medicações e demais cuidados } \\
\text { necessários; }\end{array}$ & 16 & 94,1 \\
\hline Efeitos colaterais; & 16 & 94,1 \\
\hline $\begin{array}{l}\text { Cuidados em caso de extravasamento e } \\
\text { derramamento }\end{array}$ & 16 & 94,1 \\
\hline Peculiaridades de cada quimioterápico & 17 & 100 \\
\hline \multicolumn{3}{|l|}{ Outros tópicos indicados pelos respondentes } \\
\hline $\begin{array}{l}\text { Armazenamento do quimioterápico } \\
\text { (temperatura ambiente/refrigeração) }\end{array}$ & 1 & 5,9 \\
\hline $\begin{array}{l}\text { Farmacodinâmica } \\
\text { farmacocinética/mecanismo de ação }\end{array}$ & 1 & 5,9 \\
\hline Protocolos & 1 & 5,9 \\
\hline
\end{tabular}

*Múltipla escolha

Fonte: Pesquisa direta feita pelos autores (2019)

Disponível em: https://www.nucleodoconhecimento.com.br/saude/tecnologia-educacional-emsaude 
Os enfermeiros necessitam de esclarecimentos sobre as peculiaridades de cada fármaco (100\%), e, também, sobre efeitos colaterais, cuidados com extravasamento e derramamento e pré-medicações. Esses dados apareceram em 94,1\% das respostas. O profissional de enfermagem que trabalha com pacientes oncológicos precisa ter extrema atenção e atualizar, de forma constante, os seus conhecimentos, pois cada medicação tem suas particularidades e peculiaridades, as quais nem sempre são lembradas no momento do manuseio. Informações estas foram salientadas na pesquisa realizada, enfatizando a importância de uma forma mais acessível e confiável para realizar as consultas e sanar as dúvidas, tendo como base o relato de que a rotina diária nem sempre faz com que as informações sejam gravadas, sejam elas por falta da prática rotineira ou até mesmo por falta de tempo para procurar as atualizações oferecidas pelas instituições de trabalho.

O conjunto de drogas utilizadas no tratamento, suas muitas vias de administração bem como seus efeitos colaterais, são informações pertinentes ao profissional. Afinal, além de dominá-las para realizar seu trabalho de uma forma adequada e sem intercorrências, também necessita transmiti-las aos pacientes e familiares, pois estes, ao iniciarem o tratamento quimioterápico, apresentam várias dúvidas em relação ao que será realizado (FERREIRA; MOSCATELLO, 2011). Embora tenhamos poucos estudos referentes a segurança do paciente onco pediátrico envolvendo erros na administração da terapia antineoplásica, continuamos tendo um alto potencial de danos que podem vir a ocorrer com a administração inadequada destes medicamentos.

A falta de experiência profissional, estresse, quadro de funcionários diminuto bem como em razão de prescrições incompreensíveis, são os relatos mais citados em uma entrevista feita com 160 enfermeiras da Oncology Nursing Society (ONS)[8] e as mesmas referem já terem presenciado erros de administração de antineoplásicos em seu trabalho (BELELA; PEDREIRA; PETERLINI, 2011). Na tabela 5 estão as sugestões dadas pelos respondentes sobre quais quimioterápicos são mais relevantes e que deveriam constar em um aplicativo. 
Tabela 5 - Sugestão dos enfermeiros pediátricos sobre quais quimioterápicos devem constar em uma ferramenta educativa (aplicativo) (Porto Alegre, março/2019)

\section{Cite três quimioterápicos que você considera ser mais importante constar nesta ferramenta educativa sobre administração de quimioterápicos (aplicativo).}

\begin{tabular}{|l|l|l|}
\hline Variáveis* & Número de vezes & $\%$ \\
\hline Metotrexato / MTX & 12 & 70,6 \\
\hline Ciclofosfamida & 9 & 52,9 \\
\hline Doxorrubicina & 5 & 29,4 \\
\hline Cisplatina & 5 & 29,4 \\
\hline Citarabina & 4 & 23,5 \\
\hline Vincristina & 3 & 17,6 \\
\hline Ifosfamida & 2 & 11,8 \\
\hline Carboplatina & 2 & 11,8 \\
\hline Fluorouracil & 2 & 11,8 \\
\hline Paclitaxel & 1 & 5,9 \\
\hline Aparaginase & 1 & 5,9 \\
\hline Blinatumomabe & 1 & 5,9 \\
\hline
\end{tabular}

* múltipla escolha

Fonte: Pesquisa direta feita pelos autores (2019)

A gama de medicamentos utilizados no tratamento oncológico é vasta assim como as suas categorias. Alguns são antibióticos, outros são anticorpos monoclonais que estão sendo cada vez mais utilizados neste tipo de tratamento por se tratar de uma nova tecnologia, porém os mais tradicionais ainda continuam sendo utilizados nos protocolos, pois são de extrema importância para a efetividade do conjunto das combinações de medicamentos. Os enfermeiros citaram o metotrexato $70,6 \%$ e a cisplatina $52,6 \%$ das vezes. O metotrexato, também conhecido como MTX, é uma droga que foi descoberta em 1940 e aprovada pelo FDA (Food and Drug

Disponível em: https://www.nucleodoconhecimento.com.br/saude/tecnologia-educacional-emsaude 
Administration) dos Estados Unidos da América apenas em 1953. Trata-se de um antimetabólico muito utilizado no tratamento das leucemias e foi a droga mais citada na pesquisa. É muito utilizada e apresenta diversas vias de administração que são utilizadas em diferentes etapas do tratamento, e, cada uma das suas formas de administração, apresentam diferentes possíveis reações adversas e necessitam de cuidados distintos (BONASSA; MEIRA, 2012).

\section{CONSIDERAÇÕES FINAIS}

Esta pesquisa investigou as necessidades referentes ao manuseio e aos cuidados de enfermagem na administração de quimioterápicos na pediatria. Os enfermeiros que responderam à pesquisa revelaram que sentem necessidade da criação de um mecanismo que facilite o seu dia a dia de trabalho e responda suas dúvidas com mais brevidade possível. Entretanto, eles desconhecem a existência de um aplicativo para celular que possa suprir estas necessidades. Apesar de um número limitado de respondentes, as respostas foram suficientes para observarmos que a maioria foi alocada na oncologia pediátrica logo após a conclusão do curso. Salienta-se que se trata de um setor complexo, onde é desejado um conhecimento em oncologia pediátrica maior que o conhecimento puramente acadêmico.

A cada dia, o mercado farmacêutico uma droga nova que pode ser utilizada para diferentes tratamentos bem como há a associação dessas drogas, acarretando, assim, em um infinito número de possibilidades de protocolos. Essas drogas possuem suas particularidades tanto em termos de administração quanto de efeitos colaterais. Nem sempre as orientações a tais particularidades são de fácil acesso, principalmente em um momento de intercorrência ou reação adversa. Na pediatria, as reações são muito abruptas e requerem uma ação rápida para evitar um trauma ou sequela definitiva ao paciente. As drogas mais citadas pelos profissionais foram: Metotrexato/MTX (70,6\%), Ciclofosfamida (52,9\%), Cisplatina (29,4\%), Doxorrubicina $(29,4 \%)$, Citarabina $(23,5 \%)$, dentre outras. 
Nos protocolos atuais, tais drogas nem sempre são as mais usadas, porém, são as que, frequentemente, apresentam efeitos colaterais ou outro evento durante a administração, e, por esse motivo, são as mais lembradas. O uso inadequado dessas medicações pode causar inúmeras consequências, as quais necessitam de ágil intervenção. Efeitos colaterais, administração de pré medicações, tempo de estabilidade após preparo, efeitos colaterais, armazenamento adequado bem como as peculiaridades de cada droga são algumas das situações que os enfermeiros responderam necessitar de apoio para a tomada de decisão. O enfermeiro deve dominar essas informações ou saber onde encontrá-las rapidamente para evitar essas possíveis complicações. Geralmente, as informações não são de fácil acesso para que o profissional possa fazer um diagnóstico de enfermagem rápido e evitar essas comorbidades.

Tendo em vista que, nos dias de hoje, os aplicativos para celular estão em quase todos os setores e que há uma gama variada de utilizações, podemos observar uma lacuna neste nicho de mercado. Os enfermeiros sinalizaram que um aplicativo facilitaria suas pesquisas, tornando-as mais focadas e ágeis em um momento de emergência. Dessa forma, otimizar-se-ia o pronto atendimento a fim de minimizar o trauma, o que, na maioria das vezes, pode ser grave, mas se solucionado rapidamente pode deixar pouca ou nenhuma sequela. Com base nessas pontuações, pode-se concluir que um instrumento que facilita o trabalho e cria um ambiente seguro no tratamento de pacientes pediátricos em tratamento oncológico é valido. Salienta-se, então, a importância de um aplicativo que possa auxiliar os profissionais no seu dia a dia de trabalho para este grupo de pacientes ou, quem sabe, para outras especialidades.

\section{REFERÊNCIAS}

BARRA, D. C. C. et al. Métodos para desenvolvimento de aplicativos móveis em saúde: revisão integrativa da literatura. Texto \& Contexto-Enfermagem, v. 26, n. 4, 2017. 
BELELA, A. S. C.; PEDREIRA, M. da. L. G.; PETERLINI, M. A. S. Erros de medicação em pediatria. Revista Brasileira de Enfermagem, v. 64, n. 3, p. 563-569, 2011.

BONASSA, E. A. B.; MEIRA, A. O. da. S. Neurotoxicidade. IN: BONASSA, E. M. A; GATO, M. I. R. Terapêutica Oncológica para Enfermeiros e Farmacêuticos. 4⿳a . ed. São Paulo: Editora Ateneu, 2012.

BRASIL. ANVISA. Ministério da saúde.; RESOLUÇO -RDC № 220, DE 21 DE SETEMBRO DE 2004. Brasília, 2004.

BRASIL. Ministério da saúde. Política Nacional de Gestão de Tecnologias em Saúde. Brasília, 2010.

FERREIRA, G.; MOSCATELLO, E. Assistência de Enfermagem à Criança e ao Adolescente Durante a Quimioterapia. In: MALAGUTTI, W: Oncologia Pediátrica, Uma Abordagem multiprofissional. São Paulo: Ed. Martinari, 2011.

MOTA, M. L. S.; MOLINA, P. Conceitos Gerais em Quimioterapia Antineoplásica. In: BONASSA, E. M. A; GATO, M. I. R. Terapêutica Oncológica para Enfermeiros e Farmacêuticos. 4⿳亠丷. . ed. São Paulo: Editora Ateneu, 2012.

OLIVEIRA, R. M. et al. Estratégias para promover segurança do paciente: da identificação dos riscos às práticas baseadas em evidências. Escola Anna Nery Revista de Enfermagem, v. 18, n. 1, p. 122-129, 2014.

STEFFENS, A. P. Os desafios da Saúde do Trabalhador no Cotidianos da Oncologia Pediátrica. In: MALAGUTTI, W: Oncologia Pediátrica: Uma Abordagem multiprofissional. São Paulo: Ed. Martinari, 2011.

WEGNER, W. et al. Segurança do paciente no cuidado à criança hospitalizada: evidências para enfermagem pediátrica. RGE - Revista Gaúcha de Enfermagem, v. 38, n. 1, mar. 2017. 
8. Organização sem fins lucrativos para enfermeiros e outros profissionais de saúde em atendimento ao paciente oncológico.

Enviado: Outubro, 2019.

Aprovado: Março, 2020. 